\title{
Majorization features for analytic functions involving a linear operator
}

\author{
Asena Cetinkaya ${ }^{1}$ and Oya Mert ${ }^{2}$ \\ ${ }^{1}$ İstanbul Kültür University, Department of Mathematics and Computer Science, İstanbul, Turkey \\ ${ }^{2}$ Tekirdağ Namık Kemal University, Department of Mathematics, Tekirdağ, Turkey
}

Received: 13 June 2021, Accepted: 16 June 2021

Published online: 4 July 2021.

\begin{abstract}
The first studies of majorization in univalent functions has been investigated by MacGregor in 1967. Later, several researchers studied majorization problems for univalent and multivalent functions, which are subordinate to the functions having positive real part, involving various different operators. In this paper, we define a new subclass of Ma-Minda type function class by using Carlson-Shaffer linear operator and cosine hyperbolic function. We introduce majorization properties for this function class. Moreover, some corollaries for this function class are presented.
\end{abstract}

Keywords: Analytic function, Carlson-Shaffer linear operator, Majorization, Uniformly starlike functions.

\section{Introduction}

Let $\mathcal{A}$ denote the class of analytic functions of the form

$$
f(z)=z+\sum_{n=2}^{\infty} a_{n} z^{n}
$$

in the open unit disk $\mathbb{D}=\{z \in \mathbb{C}:|z|<1\}$. Let $\mathcal{S}$ be the subclass of $\mathcal{A}$ containing all the univalent functions in $\mathbb{D}$. Denote by $\mathcal{S}^{*}$ a function $f \in \mathcal{A}$ is said to be starlike if and only if $\left(z f^{\prime}(z) / f(z)\right)>0$ for all $z \in \mathbb{D}$.

In 1991, Goodman [4] introduced the concept of uniform starlike functions in $\mathcal{S}$ denoted by UST. For $k \geq 0$, Kanas $e t$ al. [6] introduced the class of $k$-uniformly starlike functions denoted by $k$-UST. Such a class consists of functions $f \in \mathcal{A}$ that satisfy the inequality

$$
\operatorname{Re}\left(\frac{z f^{\prime}(z)}{f(z)}\right)>k\left|\frac{z f^{\prime}(z)}{f(z)}-1\right|(z \in \mathbb{D}) .
$$

We note that $1-\mathcal{U} \mathcal{S} \mathcal{T} \equiv U \mathcal{S} \mathcal{T}$ and $0-\mathcal{U S \mathcal { T }} \equiv \mathcal{S}^{*}$

Let $\Omega$ be the class of Schwarz functions $\omega$ which are analytic in $\mathbb{D}$ satisfying the conditions $\omega(0)=0$ and $|\omega(z)|<1$ for all $z \in \mathbb{D}$. If $f$ and $g$ are analytic functions in $\mathbb{D}$, then we state $f$ is subordinate to $g$, denoted by $f \prec g$, if there exists a function $\omega \in \Omega$ such that $f(z)=g(\omega(z))$ (see [3]). 
The Hadamard product (or convolution) of two functions $f$ given by (1.1) and $g(z)=z+\sum_{n=2}^{\infty} b_{n} z^{n}$ is defined by

$$
f(z) * g(z)=(f * g)(z)=z+\sum_{n=2}^{\infty} a_{n} b_{n} z^{n}
$$

Let $f$ and $g$ be analytic functions in $\mathbb{D}$, then we say that $f$ is majorized to $g$ in $\mathbb{D}$ and write $f(z) \ll g(z)(z \in \mathbb{D})$ if there exists an analytic function $\phi$ in $\mathbb{D}$ satisfying

$$
|\phi(z)| \leq 1 \text { and } f(z)=\phi(z) g(z)(z \in \mathbb{D})
$$

A majorization problem for the normalized class of starlike functions has been investigated by MacGregor in 1967 (see [7]). In 1984, Carlson and Shafffer [2] defined a linear operator $\mathcal{L}(a, c) f: \mathcal{A} \rightarrow \mathcal{A}$ given by

$$
\mathcal{L}(a, c) f(z)=\varphi(a ; c ; z) * f(z)
$$

where the incomplete beta function $\varphi(a ; c ; z)$ is defined by

$$
\varphi(a ; c ; z)=z+\sum_{n=2}^{\infty} \frac{(a)_{n-1}}{(c)_{n-1}} z^{n}, \quad\left(a \in \mathbb{R}, c \in \mathbb{R} \backslash \mathbb{Z}_{0}^{-}, \mathbb{Z}_{0}^{-}=\{0,-1, \ldots\}\right)
$$

and the Pochhammer symbol $(\sigma)_{n}$ is defined by

$$
(\sigma)_{n}=\frac{\Gamma(\sigma+n)}{\Gamma(\sigma)}= \begin{cases}1, & \text { if } n=0 \\ \sigma(\sigma+1) \ldots(\sigma+n-1), & \text { if } n \in \mathbb{N}\end{cases}
$$

In view of (1.3), the following recurrence formula can easily be obtained:

$$
z[\mathcal{L}(a, c) f(z)]^{\prime}=a \mathcal{L}(a+1, c) f(z)-(a-1) \mathcal{L}(a, c) f(z) .
$$

We note that $\mathcal{L}(a, a) f(z)=f(z)$ is the identity operator.

Ma and Minda [8] considered analytic functions $h$ with positive real part in $\mathbb{D}$ that map the disc $\mathbb{D}$ onto regions starlike with respect to 1 , symmetric with respect to real axis and normalized by $h(0)=0$ and $h^{\prime}(0)>0$, and they introduced the class

$$
\mathcal{S}^{*}(h)=\left\{f \in \mathcal{A}: \frac{z f^{\prime}(z)}{f(z)} \prec h(z), z \in \mathbb{D}\right\} .
$$

For $h(z)=(1+A z) /(1+B z),(-1 \leq B<A \leq 1)$ the class $\mathcal{S}^{*}(h)$ reduces to the family of Janowski starlike functions denoted by $\mathcal{S}^{*}(A, B)$ (see [5]). The special case $A=1-2 \gamma$ and $B=-1$ with $0 \leq \gamma<1$ leads to the class $\mathcal{S}^{*}(\gamma)$ of starlike functions of order $\gamma$. In particular, $\gamma=0$ yields the usual class $\mathcal{S}^{*}$ of starlike functions. Recently, Alotaibi et al. [1] considered the subclass $\mathcal{S}_{\text {cosh }}^{*}$ of Ma-Minda classes $\mathcal{S}^{*}(h)$ which are associated with the cosine hyperbolic function $h(z)=$ cosh that is univalent, starlike with respect to 1 and symmetric with respect to real axis.

Making use of the Carlson-Shafffer linear operator and principle of subordination, we define the following new Ma-Minda type function class related to the cosine hyperbolic function. 
Definition 1. Let $k \geq 0, a \in \mathbb{C}, c \in \mathbb{C} \backslash \mathbb{Z}_{0}^{-}$. A function $f \in \mathcal{A}$ is in the class $\mathcal{U S T}_{\cosh }(k, a, c)$ if and only if

$$
\frac{z[\mathcal{L}(a, c) f(z)]^{\prime}}{\mathcal{L}(a, c) f(z)}-k\left|\frac{z[\mathcal{L}(a, c) f(z)]^{\prime}}{\mathcal{L}(a, c) f(z)}-1\right| \prec \cosh z,
$$

where $\mathcal{L}(a, c) f$ is given by $(1.3)$ and $z \in \mathbb{D}$. For special values of parameters, we get the following classes:

(1) For $a=c=1$, we have the function class

$$
\mathcal{U S T}_{\cosh }(k):=\left\{f \in \mathcal{A}: \frac{z f^{\prime}(z)}{f(z)}-k\left|\frac{z f^{\prime}(z)}{f(z)}-1\right| \prec \cosh z\right\} .
$$

(2) For $a=c=1$ and $k=0$, we have the function class $\mathcal{S}_{\text {cosh }}^{*}$ defined in [1].

In this paper, we use a new subordination related to the cosine hyperbolic function and introduce a new subclass of univalent functions involving the Carlson-Shafffer linear operator. We obtain majorization properties and some related corollaries for this new function class.

\section{Majorization Property For The Class $U_{S \mathcal{T}_{\cosh }(k, a, c)}$}

For proving our theorems, we need the following lemma given by Nehari [9, p.168].

Lemma 1. If $\phi$ is analytic and bounded in $\mathbb{D}$, then

$$
\left|\phi^{\prime}(z)\right| \leq \frac{1-|\phi(z)|^{2}}{1-|z|^{2}}
$$

where $|z|<1$.

Theorem 1. Let $f \in \mathcal{A}$ and suppose that $g \in \mathcal{U S T}_{\cosh }(k, a, c)$ with $\mathcal{L}(a, c) f(z) \ll \mathcal{L}(a, c) g(z)$ for all $z \in \mathbb{D}$. Then, for $|z| \leq r_{1}$ we have

$$
|\mathcal{L}(a+1, c) f(z)| \leq|\mathscr{L}(a+1, c) g(z)|
$$

where $r_{1}$ is the smallest positive root of the equation

$$
\left(1-r^{2}\right)(\cos r+|a|(1+k)-1)-2 r(1+k)=0
$$

Proof Since $g \in \mathcal{U S T}_{\text {cosh }}(k, a, c)$, then from (1.5) we write

$$
\frac{z[\mathcal{L}(a, c) g(z)]^{\prime}}{\mathcal{L}(a, c) g(z)}-k\left|\frac{z[\mathcal{L}(a, c) g(z)]^{\prime}}{\mathcal{L}(a, c) g(z)}-1\right|=\cosh \omega(z),
$$

where $\omega$ is a Schwarz function in $\mathbb{D}$ satisfying $\omega(0)=0$ and $|\omega(z)| \leq|z|$. Letting

$$
\eta=\frac{z[\mathcal{L}(a, c) g(z)]^{\prime}}{\mathcal{L}(a, c) g(z)}
$$

then (2.3) can be written as

$$
\eta-k|\eta-1|=\cosh \omega(z)
$$


which leads to

$$
\eta=\frac{\cosh \omega(z)-k e^{-i \theta}}{1-k e^{-i \theta}} \quad(\theta \in \mathbb{R})
$$

Substituting (2.4) into (2.5), using (1.4) and by applying the condition $|\omega(z)| \leq|z|$, we arrive at

$$
|\mathcal{L}(a, c) g(z)| \leq \frac{|a|(1+k)}{|\cosh \omega(z)|+|a|(1+k)-1}|\mathcal{L}(a+1, c) g(z)| .
$$

Because $\mathcal{L}(a, c) f$ is majorized by $\mathcal{L}(a, c) g$, there exists an analytic function $\phi$ given by (1.2) in $\mathbb{D}$ with $|\phi(z)| \leq 1$ satisfying

$$
\mathcal{L}(a, c) f(z)=\phi(z) \mathcal{L}(a, c) g(z)
$$

Differentiating on both sides of (2.7) with respect to $z$ and multiplying by $z$, we obtain

$$
z[\mathcal{L}(a, c) f(z)]^{\prime}=z \phi^{\prime}(z) \mathcal{L}(a, c) g(z)+z \phi(z)[\mathcal{L}(a, c) g(z)]^{\prime}
$$

By using (1.4) and (2.7) in (2.8), we get

$$
\mathcal{L}(a+1, c) f(z)=\frac{1}{a} z \phi^{\prime}(z) \mathcal{L}(a, c) g(z)+\phi(z) \mathcal{L}(a+1, c) g(z)
$$

Substituting (2.6) into (2.9) and applying Lemma 1, we get

$$
|\mathcal{L}(a+1, c) f(z)| \leq\left[\frac{|z|\left(1-|\phi(z)|^{2}\right)}{\left(1-|z|^{2}\right)} \frac{(1+k)}{|\cosh \omega(z)|+|a|(1+k)-1}+\phi(z)\right]|\mathcal{L}(a+1, c) g(z)|,
$$

Let $\omega(z)=R e^{i t}$ with $R \leq|z|=r,-\pi \leq t \leq \pi$. A computation shows that

$$
\left.\left.\left|\cosh \left(R e^{i t}\right)\right|^{2}=\cosh ^{2}(R \cos t) \cos ^{2}(R \sin t)\right)+\sinh ^{2}(R \cos t) \sin ^{2}(R \sin t)\right]^{2}=: \Psi(t) .
$$

It is easy to evaluate that $\Psi^{\prime}(t)=0$ has five roots as $0, \mp \pi$ and $\mp \pi / 2$ in $[-\pi, \pi]$. Since $\Psi(t)$ is symmetric with respect to real axis, it is sufficent to consider the roots in $[0, \pi]$. It follows from

$$
\max \{\Psi(0), \Psi(\pi)\}=\cosh ^{2} R, \quad \min \{\Psi(\pi / 2)\}=\cos ^{2} R
$$

that

$$
\cos r \leq \cos R \leq|\cosh \omega(z)| \leq \cosh R \leq \cosh r
$$

Upon setting $|z|=r$ and $|\phi(z)|=\rho \quad(0 \leq \rho \leq 1)$, the inequality (2.10) can be written as

$$
|\mathcal{L}(a+1, c) f(z)| \leq \Theta(r, \rho)|\mathcal{L}(a+1, c) g(z)|
$$

where

$$
\Theta(r, \rho)=\frac{r\left(1-\rho^{2}\right)}{\left(1-r^{2}\right)} \frac{1+k}{\cos r+|a|(1+k)-1}+\rho .
$$

In order to determine $r_{1}$, we choose

$$
r_{1}=\max \{r \in(0,1): \Theta(r, \rho) \leq 1, \forall \rho \in[0,1]\}=\max \{r \in(0,1): \chi(r, \rho) \geq 0, \forall \rho \in[0,1]\}
$$


where

$$
\chi(r, \rho)=\left(1-r^{2}\right)(\cos r+|a|(1+k)-1)-r(1+\rho)(1+k) .
$$

Since $\frac{\partial}{\partial \rho} \chi(r, \rho)=-r(1+k)<0$, therefore $\chi(r, \rho)$ takes its minimum for $\rho=1$, namely

$$
\min \{\chi(r, \rho) \geq 0, \rho \in[0,1]\}=\chi(r, 1)=: \chi(r)
$$

where

$$
\chi(r)=\left(1-r^{2}\right)(\cos r+|a|(1+k)-1)-2 r(1+k) .
$$

Moreover $\chi(0)=|a|(1+k) \geq 0$ and $\chi(1)=-2(1+k)<0$, thus there exists $|z| \leq r_{1}$ such that $\chi(r) \geq 0$ for all $r \in\left[0, r_{1}\right]$, where $r_{1}$ is the smallest positive root of the equation (2.2). This completes the proof.

Corollary 1. If $f \in \mathcal{A}$ and $g \in \mathcal{U S \mathcal { T }} \mathcal{T}_{\cosh }(k)$ with $f(z) \ll g(z)$ for all $z \in \mathbb{D}$. Then, for $|z| \leq r_{2}$ we have $\left|f^{\prime}(z)\right| \leq\left|g^{\prime}(z)\right|$, where $r_{2}$ is the smallest positive root of the equation

$$
\left(1-r^{2}\right)(\cos r+k)-2 r(1+k)=0 .
$$

Corollary 2. If $f \in \mathcal{A}$ and $g \in \mathcal{S}_{\text {cosh }}^{*}$ with $f(z) \ll g(z)$ for all $z \in \mathbb{D}$. Then, for $|z| \leq r_{3}$ we have $\left|f^{\prime}(z)\right| \leq\left|g^{\prime}(z)\right|$, where $r_{3}$ is the smallest positive root of the equation

$$
\left(1-r^{2}\right) \cos r-2 r=0 .
$$

\section{Competing interests}

The authors declare that they have no competing interests.

\section{Authors' contributions}

All authors have contributed to all parts of the article. All authors read and approved the final manuscript.

\section{References}

[1] A. Alotaibi, M. Arif, M.A. Alghamdi and S. Hussain, Starlikness associated with Cosine Hyperbolic function, Mathematics, 8(7) (2020), 1118.

[2] B.C. Carlson and D.B. Shaffer, Starlike and prestarlike hypergeometric functions, SIAM J. Math. Anal. 15 (1984), $737-745$.

[3] P.L. Duren, Univalent Functions, Springer, New York, 1983.

[4] A.W. Goodman, On uniformly convex functions, Ann. Polon. Math. 56 (1991), 87-92.

[5] W. Janowski, Some extremal problems for certain families of analytic functions I, Ann. Polon. Math. 28 (1973), $297-326$.

[6] S. Kanas and A. Wiśniowska, Conic domains and starlike functions, Rev. Roumaine Math. Pures Appl. 45 (2000), $647-657$.

[7] T.H. MacGregor, Majorization by univalent function, Duke Math. J. 34(1) (1967), 95-102.

[8] W.C. Ma and D. Minda, A unified treatment of some special classes of univalent functions, In: Proceedings of the Conference on Complex Analysis, Tianjin, (1992), 157-169.

[9] Z. Nehari, Conformal mappings, McGraw-Hill Book Company, New York, 1952. 